\title{
pth Moment Exponential Stability of Stochastic PWM Feedback Systems with Time-Varying Delays
}

\author{
Zhong Zhang and Lixia Ye \\ College of Mathematics and Statistics, Chongqing University, Chongqing 400044, China \\ Correspondence should be addressed to Zhong Zhang, zhanguicqu@gmail.com
}

Received 22 September 2012; Accepted 12 November 2012

Academic Editor: Chuandong Li

Copyright (C) 2012 Z. Zhang and L. Ye. This is an open access article distributed under the Creative Commons Attribution License, which permits unrestricted use, distribution, and reproduction in any medium, provided the original work is properly cited.

This paper further studies the $p$ th moment exponential stability of stochastic pulse-widthmodulated (PWM) feedback systems with distributed time-varying delays. We establish several globally exponential stability criteria for such PWM feedback systems by using LyapunovKrasovskii functional and then present an upper bound of the parameter of PWM when the system is stable and such system has stronger anti-interference performance than the system without time-varying delays. Furthermore, we present two examples to show the effectiveness and conservativeness of the theoretical results.

\section{Introduction}

Pulse-width modulation has extensively been used in attitude control systems, adaptive control systems, signal processing, power control systems, modeling of neuron behavior, and the like (e.g., see [1-5]). In many areas, especially engineering applications, how to keep the scheduled operation or work of the state counts for much. Therefore, there has been a growing research interest on the stability analysis for PWM feedback systems, and a set of stability results have been established by a variety of methods [6-22]. In the actual process, however, it is always operated in all kinds of accidental or continued disturbances. Time delay will inevitably occur in electronic neural networks owing to the unavoidable finite switching speed of amplifiers. In recent years, the stability analysis of stochastic systems, especially the systems with time delay, is interesting to many investigators, and many results of stability criteria of these systems have been reported [15-22].

There are, however, only a few results concerning the qualitative properties of stochastic impulsive systems with time-varying delays. In [15], the authors investigated robust exponential stability and delayed-state-feedback stabilization of uncertain impulsive 
stochastic systems with time-varying delays. Besides, Sun and Cao [17] give some definitions on the $p$ th moment exponential stability in mean and established several $p$ th moment globally stability criteria in mean. In [12, 13], Hou and Michel established new Lyapunov and Lagrange stability results for pulse-width-modulation (PWM) feedback systems subjected to random disturbance.

To the best of the authors' knowledge, there are few (if any) results for the stability analysis of stochastic PWM systems with time-varying delays. Based on the pulse-widthmodulation feedback system uniqueness, obviously, such system subjected to random disturbance and time-varying delays is somewhat complex in comparison with most of the systems in the literature. It is noted that the linear plant considered herein is Hurwitz stable, that is, all the eigenvalues of the linear plant are in the left side of the complex plane. In the present paper, we try to make the contribution on this issue. By choosing reasonable Lyapunov-Krasovskii functional, combined with linear matrix inequalities and Itô integration method, we will establish several Lyapunov and Lagrange criteria for $p$ th moment exponential stability in mean and then present an algorithm to compute the upper bound for the parameters of PWM. We will characterize the relationship among the parameters of pulsewidth modulation, time-varying delays, and the coefficient of state vectors of the feedback systems. It will be shown that when the random disturbance is sufficiently small such PWM feedback system is $p$ th moment exponentially stable in mean provided that the upper bounds of parameters of pulse-width modulator are selected properly. We also demonstrate that such system has the stronger anti-interference performance and tending to the equilibrium point speed more quickly by means of two numerical examples.

\section{Notations and Some Definitions}

Let $(\Omega, F, P)$ denote the underlying probability space for all the systems that will be considered, where $\Omega$ is the sample space, $F$ is the $\sigma$-algebra of subsets of the sample space, and $P$ is the probability measure. An $R^{n}$-valued random variable $x$ with domain $X$ is a measurable function from $\Omega$ to $X \subset R^{n}$. A family $\{x(t), t \in I\}$ of $R^{n}$ valued random variables with domain $X$ defined on a probability space $(\Omega, F, P)$ is called a stochastic process with index set $I$ and state space $\left(X, F^{n}\right)$.

Definition 2.1. Let $(X, d)$ be a metric space, $X \subset R^{n}, A \subset X$, and let $T \subset R^{+}$. For any fixed $a \in A$ ( $a$ is called the initial state), $t_{0} \in T$, a stochastic process $\left\{x\left(t, \omega, a, t_{0}\right), t \in T_{a, t_{0}}\right\}$ with domain $X$ is called a stochastic motion if $x\left(t_{0}, \omega, a, t_{0}\right)=a$ for all $\omega \in \Omega$, where $T_{a, t_{0}}=\left[t_{0}, t_{1}\right) \cap T, t_{1}>t_{0}$, and $t_{1}$ is finite or infinite.

Definition 2.2. Let $S$ be a family of stochastic motions with domain $X$ given by

$$
S \subset\left\{x\left(\cdot, \cdot, a, t_{0}\right): x\left(t_{0}, \omega, a, t_{0}\right)=a, \omega \in \Omega, a \in A, t_{0} \in T\right\} .
$$

We call the four-tuple $\{T, X, A, S\}$ a stochastic dynamical system.

Definition 2.3. Let $\{T, X, A, S\}$ be a stochastic dynamical system. A set $M \subset A$ is said to be invariant with respect to system $S$ (or short, $(S, M)$ is invariant) if $a \in M$ implies that $P\{\omega$ : $x\left(t, \omega, a, t_{0}\right) \in M$ for all $\left.t \in T_{a, t_{0}}\right\}=1$, for all $t_{0} \in T$ and all $x\left(\cdot, \cdot, a, t_{0}\right) \in S$. 


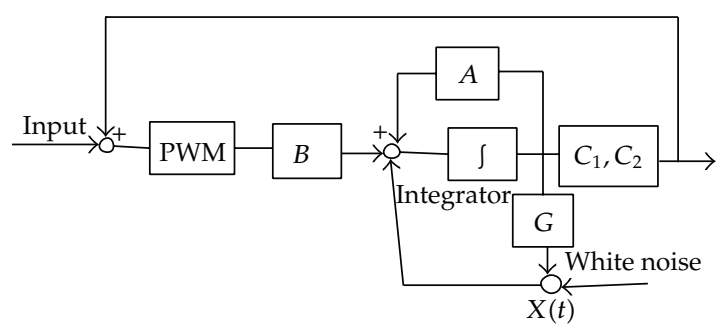

Figure 1: Block diagram of stochastic PWM feedback systems with time-varying delays.

Definition 2.4. $x_{0} \in A$ is called an equilibrium point of a stochastic dynamical system $\{T, X, A, S\}$ if the set $\left\{x_{0}\right\}$ is invariant with respect to $S$.

Definition 2.5. Let $\{T, X, A, S\}$ be a stochastic dynamical system, and let $d$ be the metric on $X$. A set $M \subset A$ is said to be the $p$ th moment exponentially stable in mean (i.e., $(S, M)$ is said to be the $p$ th moment exponentially stable in mean) if for any $t_{0} \in T$, there exists a $\delta=\delta\left(t_{0}, \varepsilon\right)$ and constants $\beta>0, k>1$ such that $E\left[d\left(x\left(t, \omega, a, t_{0}\right), M\right)^{p}\right]<k\|a\|^{p} e^{-\beta\left(t-t_{0}\right)}$ for any process $x\left(\cdot, \cdot, a, t_{0}\right) \in S$, whenever $d(a, M)<\delta$, where $a$ is called the initial state and $E[\cdot]$ denotes the expectation of a random process. If $\delta$ is independent of $t_{0},(S, M)$ is said to be the pth moment uniformly exponentially stable in mean. $(S, M)$ is said to be the pth moment uniformly asymptotically exponentially stable in mean if it is uniformly stable in the $p$ th mean and if there exists $\delta>0, \tau>0$ and constants $\beta>0, k>1$ such that for any process $x\left(\cdot, \cdot, a, t_{0}\right) \in S$, whenever $d(a, M)<\delta$ implies that $E\left[d\left(x\left(t, \omega, a, t_{0}\right), M\right)^{p}\right]<k\|a\|^{p} e^{-\beta\left(t-t_{0}\right)}$ for all $t>t_{0}+\tau$.

\section{Main Results}

The PWM feedback system to be considered in this paper is shown in Figure 1.

The pulse-width modulator is described by

$$
u(t)=m(e(t))= \begin{cases}M \operatorname{sgn}(e(k T)) & t \in\left[k T, k T+T_{k}\right), \\ 0 & t \in\left[k T+T_{k}, k T+T\right),\end{cases}
$$

where $e(t)=r(t)-y(t)$ with $r(t)$ being the external input and $y(t)$ the system output, and, for $k=0,1,2, \ldots$, the pulse-width $T_{k}$ and the sign function sgn are given, respectively, by

$$
\begin{gathered}
T_{k}= \begin{cases}\beta|e(k T)|, & |e(k T)| \leq \frac{T}{\beta^{\prime}} \\
T, & |e(k T)|>\frac{T}{\beta^{\prime}}\end{cases} \\
\operatorname{sgn}(\sigma)= \begin{cases}1 & \sigma>0 \\
0 & \sigma=0 \\
-1 & \sigma<0\end{cases}
\end{gathered}
$$


The sampling period $T$, the amplitude of the pulse $M$, and $\beta$ are all assumed to be constants. And throughout this paper, we always assume that $r(t) \equiv 0$. Under these assumptions, the PWM feedback system with the output function can be described by

$$
\begin{gathered}
d x(t)=A x(t) d t+A_{d} x(t-d) d t+B u(t) d t+G x(t) d W_{t}, \\
y(t)=C_{1} x(t)+C_{2} x(t-d),
\end{gathered}
$$

where $x \in R^{n}, y \in R, u \in R$, is output of the pulse-width modulator, $A, A_{d}, B, C_{1}, C_{2}$, and $G$ are matrices of appropriate dimensions, $d=\left(d_{i}\right) \in R^{n}, 0<d_{i} \leq T-T_{k}, i=1,2, \ldots, n$, and $W(t)$ is a scalar wiener process.

Note that $\left(x_{1}^{T}, x_{2}\right)=\left(0^{T}, 0\right)^{T}$ is an equilibrium point of PWM feedback system (3.3).

Lemma 3.1 (Schur complement). Given the matrix $S=\left(\begin{array}{ll}S_{11} & S_{12} \\ S_{21} & S_{22}\end{array}\right)$. Then $S<0$ is equivalent to any one of the following conditions:

(i) $S_{11}<0, S_{22}-S_{21} S_{11}^{-1} S_{12}<0$,

(ii) $S_{22}<0, S_{11}-S_{12} S_{22}^{-1} S_{21}<0$.

Lemma 3.2 (the Itô isometry). Assume $W(t)$ is a scalar wiener process. If $\phi(t, \omega)$ is bounded and elementary, then

$$
E\left[\left(\int_{S}^{T} \phi(t, \omega) d W_{t}\right)^{2}\right]=E\left[\int_{S}^{T} \phi(t, \omega)^{2} d t\right]
$$

Proof. Put $\Delta W_{j}=W_{t_{j+1}}-W_{t_{j}}$, then

$$
E\left[e_{i} e_{j} \Delta W_{i} \Delta W_{j}\right]= \begin{cases}0 & \text { if } i \neq j, \\ E\left[e_{j}^{2}\right]\left(t_{j+1}-t_{j}\right) & \text { if } i=j,\end{cases}
$$

using that $e_{i} e_{j} \Delta W_{i}$ and $\Delta W_{j}$ are independent if $i<j$. Thus

$$
\begin{aligned}
E\left[\left(\int_{S}^{T} \phi(t, \omega) d W_{t}\right)^{2}\right] & =\sum_{i, j} E\left[e_{i} e_{j} \Delta W_{i} \Delta W_{j}\right] \\
& =\sum_{j} e_{j}^{2} \cdot\left(t_{i}-t_{j}\right) \\
& =E\left[\int_{S}^{T} \phi(t, \omega)^{2} d t\right] .
\end{aligned}
$$

Theorem 3.3. Assume that the matrix $A$ in (3.3) is Hurwitz stable. Then the equilibrium $x_{e}=0$ of PWM feedback system (3.3) is the pth $\left(p \in Z^{+}\right)$moment uniformly exponentially stable in mean in the large provided that the following conditions are satisfied: 
(i)

$$
M \beta<\inf _{\tau_{k} \in[0, \infty)} \frac{\lambda_{m}\left(\Gamma_{1}\right)+\sqrt{\lambda_{m}^{2}\left(\Gamma_{1}\right)+4 \lambda_{M}\left(\Gamma_{2}\right)}}{2 \lambda_{M}\left(\Gamma_{2}\right)},
$$

where

$$
\begin{aligned}
& \Gamma_{1}=U_{1}\left(\tau_{k}\right) C_{1}+\left(U_{1}\left(\tau_{k}\right) C_{1}\right)^{T}, \\
& \Gamma_{2}=C_{1}^{T}\left(G_{1}\left(\tau_{k}\right)+G_{2}\left(\tau_{k}\right)\right) C_{1}
\end{aligned}
$$

with

$$
\begin{gathered}
G_{i}\left(T_{k}\right)=W\left(T_{k}\right)^{T}\left(P_{i}-I\right) W\left(T_{k}\right) \\
U_{i}\left(T_{k}\right)=\left(P_{i}-I\right) W\left(T_{k}\right), \quad i=1,2
\end{gathered}
$$

(ii)

$$
\varphi_{22}-\varphi_{12} \varphi_{11}^{-1} \varphi_{12}^{T}<0
$$

where

$$
\begin{aligned}
\varphi_{11}= & -I-M \beta \Gamma_{1}+M^{2} \beta^{2} \Gamma_{2}, \\
\varphi_{12}= & -M \beta\left[U_{1}\left(\tau_{k}\right) C_{2}+\left(U_{2}\left(\tau_{k}\right) C_{1}\right)^{T} e^{A d}\right] \\
& +M^{2} \beta^{2} C_{1}^{T}\left(G_{1}\left(\tau_{k}\right)+G_{2}\left(\tau_{k}\right)\right) C_{2}, \\
\varphi_{22}= & \left(e^{A T}\right)^{T} P_{2} e^{A T}-P_{2}-M \beta\left[\left(U_{2}\left(\tau_{k}\right) C_{2}\right)^{T} e^{A d}+\left(e^{A d}\right)^{T} U_{2}\left(\tau_{k}\right) C_{2}\right] \\
& +M^{2} \beta^{2} C_{2}^{T}\left(G_{1}\left(\tau_{k}\right)+G_{2}\left(\tau_{k}\right)\right) C_{2},
\end{aligned}
$$

(iii) there exists a constant $\delta>0$, such that, whenever $\|G\|<\delta$,

$$
\max \left\{\left\|A_{d}\right\|^{2},\|G\|^{2}\right\} \cdot e^{\left(\left\|A_{d}\right\|^{2}+\|G\|^{2}\right) T}<\mu,
$$

where $\mu$ is scalar satisfying

$$
c(\mu)=1-2\|\Phi\| \sqrt{\mu}-4\|P\| \mu>0
$$


with

$$
\Phi=\left(\begin{array}{cc}
P_{1} e^{A T}\left(I-M \beta W\left(\tau_{k}\right) C_{1}\right) & -M \beta P_{1} e^{A T} W\left(\tau_{k}\right) C_{2} \\
-M \beta P_{2} e^{A(T-d)} W\left(\tau_{k}\right) C_{1} & P_{2} e^{A(T-d)}\left(e^{A d}-M \beta W\left(\tau_{k}\right) C_{2}\right)
\end{array}\right)
$$

where $P_{1}=P_{1}^{T}>0, P_{2}=P_{2}^{T}>0$ satisfying

$$
\begin{gathered}
\left(e^{A T}\right)^{T} P_{1} e^{A T}-P_{1}=-I, \\
\left(e^{A(T-d)}\right)^{T} P_{2} e^{A(T-d)}-P_{2}=-I .
\end{gathered}
$$

Proof. Since $\left(E\|X\|^{p}\right)^{1 / p}$ is nondecreasing in $p$, the equilibrium $x_{e}=0$ is stable (resp., asymptotically stable, etc.) implying that it is the $q$ th moment stable (resp., asymptotically stable, etc.) in mean for all $q<p$. Firstly, we will provide to prove the theorem for even integers.

Integrating (3.3), we have

$$
\begin{aligned}
x(t)= & e^{A(t-k T)} x(k T)+\int_{k T}^{t} e^{A(t-s)} A_{d} x(s-d) d s \\
& +\int_{k T}^{t} e^{A(t-s)} B u(s) d s+\int_{k T}^{t} e^{A(t-s)} G x(s) d W_{s} .
\end{aligned}
$$

Therefore, when $t=k T+T$,

$$
\begin{aligned}
x(k T+T)= & e^{A T} x(k T)+\int_{k T}^{k T+T} e^{A(k T+T-s)} B u(s) d s \\
& +\int_{k T}^{k T+T} e^{A(k T+T-s)} A_{d} x(s-d) d s+\int_{k T}^{k T+T} e^{A(k T+T-s)} G x(s) d W_{s} \\
= & e^{A T} x(k T)-\int_{k T}^{k T+T_{k}} e^{A(k T+T-s)} B M \operatorname{sgn}\left(C_{1} x(k T)+C_{2} x(k T-d)\right) d s \\
& +\int_{k T}^{k T+T} e^{A(k T+T-s)} A_{d} x(s-d) d s+\int_{k T}^{k T+T} e^{A(k T+T-s)} G x(s) d W_{s} \\
= & e^{A T}\left[\left(I-M \beta W\left(\tau_{k}\right) C_{1}\right) x(k T)-M \beta W\left(\tau_{k}\right) C_{2} x(k T-d)\right] \\
& +\int_{k T-d}^{k T+T-d} e^{A(k T+T-d-s)} A_{d} x(s) d s+\int_{k T}^{k T+T} e^{A(k T+T-s)} G x(s) d W_{s} .
\end{aligned}
$$


For $t=k T+T-d$, we have

$$
\begin{aligned}
& x(k T+T-d)=e^{A T} x(k T-d)+\int_{k T-d}^{k T+T-d} e^{A(k T+T-d-s)} B u(s) d s \\
& +\int_{k T-d}^{k T+T-d} e^{A(k T+T-d-s)} A_{d} x(s-d) d s+\int_{k T-d}^{k T+T-d} e^{A(k T+T-d-s)} G x(s) d W_{s} \\
& =e^{A T} x(k T-d)-\int_{k T}^{k T+T_{k}} e^{A(k T+T-d-s)} B M \operatorname{sgn}\left(C_{1} x(k T)+C_{2} x(k T-d)\right) d s \\
& +\int_{k T-d}^{k T+T-d} e^{A(k T+T-d-s)} A_{d} x(s-d) d s+\int_{k T-d}^{k T+T-d} e^{A(k T+T-d-s)} G x(s) d W_{s} \\
& =e^{A(T-d)}\left[-M \beta W\left(\tau_{k}\right) C_{1} x(k T)+\left(e^{A d}-M \beta W\left(\tau_{k}\right) C_{2}\right) x(k T-d)\right] \\
& +\int_{k T-d}^{k T+T-d} e^{A(k T+T-d-s)} A_{d} x(s-d) d s+\int_{k T}^{k T+T} e^{A(k T+T-s)} G x(s-d) d W_{s}
\end{aligned}
$$

with

$$
\begin{gathered}
\tau_{k}=\beta\left|C_{1} x(k T)+C_{2} x(k T-d)\right| \\
= \begin{cases}T_{k}, & T_{k}<T, \\
\geq T, & T_{k}=T,\end{cases} \\
W\left(\tau_{k}\right)= \begin{cases}0, & \tau_{k}=0, \\
\frac{I-e^{-A T_{k}}}{T_{k}} A^{-1} B, & \tau_{k}<T, \\
\frac{I-e^{-A T}}{\tau_{k}} A^{-1} B=\frac{T}{\tau_{k}} W(T), & \tau_{k} \geq T .\end{cases}
\end{gathered}
$$

To simplify our notations, let

$$
\begin{aligned}
H_{1} & =\left[I-M \beta W\left(\tau_{k}\right) C_{1}-M \beta W\left(\tau_{k}\right) C_{2}\right], \\
H_{2} & =\left[-M \beta W\left(\tau_{k}\right) C_{1} e^{A d}-M \beta W\left(\tau_{k}\right) C_{2}\right], \\
h(k T) & =\int_{k T-d}^{k T+T-d} e^{A(k T+T-d-s)} A_{d} x(s) d s, \\
h(k T-d) & =\int_{k T-d}^{k T+T-d} e^{A(k T+T-d-s)} A_{d} x(s-d) d s,
\end{aligned}
$$




$$
\begin{aligned}
\tilde{h}(k T) & =\int_{k T}^{k T+T} e^{A(k T+T-s)} G x(s) d W_{s}, \\
\tilde{h}(k T-d) & =\int_{k T}^{k T+T} e^{A(k T+T-s)} G x(s-d) d W_{s}, \\
X(k T) & =\left[x^{T}(k T), x^{T}(k T-d)\right]^{T} .
\end{aligned}
$$

Then, (3.17) and (3.18) are reduced to

$$
\begin{gathered}
x(k T+T)=e^{A T} H_{1} X(k T)+h(k T)+\tilde{h}(k T), \\
x(k T+T-d)=e^{A(T-d)} H_{2} X(k T-d)+h(k T-d)+\tilde{h}(k T-d) .
\end{gathered}
$$

Choosing the quadratic Lyapunov-Krasovskii functional $V: R^{n} \rightarrow R^{+}$,

$$
V(x)=x^{T}(t) P_{1} x(t)+x^{T}(t-d) P_{2} x(t-d) .
$$

Then,

$$
\begin{aligned}
\nabla E[V(x)]= & E[V(x(k T+T))]-E[V(x(k T))] \\
& -E[V(x(k T))]-E[V(x(k T-d))] \\
= & E\left[x^{T}(k T+T) P_{1} x(k T+T)\right]+E\left[x^{T}(k T+T-d) P_{2} x(k T+T-d)\right] \\
& -E\left[x^{T}(k T) P_{1} x(k T)\right]-E\left[x(k T-d) P_{2} x(k T-d)\right] \\
= & E\left[X^{T}(k T)\left(H_{1}^{T}\left(P_{1}-I\right) H_{1}+H_{2}^{T}\left(P_{2}-I\right) H_{2}-P\right) X(k T)\right] \\
& +2 E\left[(h(k T)+\tilde{h}(k T))^{T} P_{1} e^{A T} H_{1} X(k T)\right] \\
& +2 E\left[(h(k T-d)+\tilde{h}(k T-d))^{T} P_{2} e^{A(T-d)} H_{2} X(k T)\right] \\
& +E\left[(h(k T)+\tilde{h}(k T))^{T} P_{1}(h(k T)+\tilde{h}(k T))\right] \\
& +E\left[(h(k T-d)+\tilde{h}(k T-d))^{T} P_{2}(h(k T-d)+\tilde{h}(k T-d))\right] \\
= & E\left[X^{T}(k T)\left(H_{1}^{T}\left(P_{1}-I\right) H_{1}+H_{2}^{T}\left(P_{2}-I\right) H_{2}-P\right) X(k T)\right] \\
& +2 E\left[Z^{T}(k T) \Phi X(k T)\right]+2 E\left[\tilde{Z}^{T}(k T) \Phi X(k T)\right] \\
& +E\left[Z^{T}(k T) P Z(k T)\right]+2 E\left[Z^{T}(k T) P \tilde{Z}(k T)\right]+E\left[\tilde{Z}^{T}(k T) P \tilde{Z}(k T)\right],
\end{aligned}
$$


where

$$
\begin{aligned}
& Z(k T)=\left(h^{T}(k T) h^{T}(k T-d)\right)^{T}, \\
& \tilde{Z}(k T)=\left(\begin{array}{ll}
\tilde{h}^{T}(k T) & \left.\tilde{h}^{T}(k T-d)\right)^{T},
\end{array}\right. \\
& \Phi=\left(\begin{array}{c}
P_{1} e^{A T} H_{1} \\
P_{2} e^{A(T-d)} H_{2}
\end{array}\right)=\left(\begin{array}{cc}
P_{1} e^{A T}\left(I-M \beta W\left(\tau_{k}\right) C_{1}\right) & -M \beta P_{1} e^{A T} W\left(\tau_{k}\right) C_{2} \\
-M \beta P_{2} e^{A(T-d)} W\left(\tau_{k}\right) C_{1} & P_{2} e^{A(T-d)}\left(e^{A d}-M \beta W\left(\tau_{k}\right) C_{2}\right)
\end{array}\right), \\
& H=H_{1}^{T}\left(P_{1}-I\right) H_{1}+H_{2}^{T}\left(P_{2}-I\right) H_{2}-P=\left(\begin{array}{cc}
\varphi_{11} & \varphi_{12} \\
\varphi_{12}^{T} & \varphi_{22}
\end{array}\right) \text {. }
\end{aligned}
$$

Then (3.23) is reduced to

$$
\begin{aligned}
\nabla E[V(x)]= & E\left[X^{T}(k T) H X(k T)\right]+2 E\left[Z^{T}(k T) \Phi X(k T)\right]+2 E\left[\tilde{Z}^{T}(k T) \Phi X(k T)\right] \\
& +E\left[Z^{T}(k T) P Z(k T)\right]+2 E\left[Z^{T}(k T) P \tilde{Z}(k T)\right]+E\left[\tilde{Z}^{T}(k T) P \tilde{Z}(k T)\right] .
\end{aligned}
$$

Let $\lambda_{m}(\cdot)$ and $\lambda_{M}(\cdot)$ denote the minimum and maximum eigenvalues of a matrix, respectively. By Lemma 3.1, it is obvious that $H$ is a negative definite matrix if and only if $\varphi_{11}<0$ and $\varphi_{22}-\varphi_{12} \varphi_{11}^{-1} \varphi_{12}^{T}<0$. Noting that

$$
\begin{aligned}
\lambda_{M}\left(\varphi_{11}\right) \leq & -1-M \beta \lambda_{m}\left[U_{1}\left(\tau_{k}\right) C_{1}+\left(U_{1}\left(\tau_{k}\right) C_{1}\right)^{T}\right] \\
& +M^{2} \beta^{2} \lambda_{M}\left[C_{1}^{T}\left(G_{1}\left(\tau_{k}\right)+G_{2}\left(\tau_{k}\right)\right) C_{1}\right] \\
\triangleq & \Theta_{M \beta} .
\end{aligned}
$$

From condition (i) it follows that $\Theta_{M \beta}<0$, and hence the claim that $\varphi_{11}<0$ is true. From condition (ii) we then conclude that $H$ is a negative definite matrix based on Lemma 3.1.

Now we establish an estimation on $Z(k T)$ and $\tilde{Z}(k T)$. Let $\mu>0$ be arbitrary. We will show that there exists $\delta>0$ whenever $\|G\|<\delta$, such that

$$
E\left[\|Z(k T)\|^{2}\right]<\mu E\left[\|X(k T)\|^{2}\right], \quad E\left[\|\tilde{Z}(k T)\|^{2}\right]<\mu E\left[\|X(k T)\|^{2}\right] .
$$


For $t \in[k T, k T+T]$, we have

$$
\begin{aligned}
x(t)= & x(k T)+\int_{k T}^{t} A x(s) d s+\int_{k T}^{t} A_{d} x(s-d) d s \\
& +\int_{k T}^{t} B u(s) d s+\int_{k T}^{t} G x(s) d W_{s} \\
= & x(k T)+\int_{k T}^{t} A x(s) d s+\int_{k T-d}^{t-d} A_{d} x(s) d s \\
& +\int_{k T}^{t} B u(s) d s+\int_{k T}^{t} G x(s) d W_{s} \\
x(t-d)= & x(k T-d)+\int_{k T-d}^{t-d} A x(s) d s+\int_{k T-d}^{t-d} A_{d} x(s-d) d s \\
& +\int_{k T-d}^{t-d} B u(s) d s+\int_{k T-d}^{t-d} G x(s) d W_{s} \\
& +\int_{k T-d}^{t-d} B u(s) d s+\int_{k T}^{t} G x(s-d) d W_{s} . \\
= & (k T-d)+\int_{k T}^{t} A x(s-d) d s+\int_{k T-d}^{t-d} A_{d} x(s-d) d s \\
& \\
& \\
& \\
&
\end{aligned}
$$

Then, we have

$$
\begin{aligned}
X(t)= & X(k T)+L(k T)+\int_{k T}^{t} \tilde{A} X(s) d s \\
& +\int_{k T-d}^{t-d} \tilde{A}_{d} X(s) d s+\int_{k T}^{t} \tilde{G} X(s) d W_{s},
\end{aligned}
$$

where

$$
\begin{gathered}
\tilde{A}=\left(\begin{array}{cc}
A & 0 \\
0 & A
\end{array}\right), \quad \tilde{A}_{d}=\left(\begin{array}{cc}
A_{d} & 0 \\
0 & A_{d}
\end{array}\right), \quad \tilde{G}=\left(\begin{array}{cc}
G & 0 \\
0 & G
\end{array}\right), \\
L(k T)=\left(\begin{array}{cc}
\int_{k T}^{t} B u(s) d s & 0 \\
0 & \int_{k T-d}^{t-d} B u(s) d s
\end{array}\right) .
\end{gathered}
$$


Similarly, we have

$$
\begin{aligned}
& Z(k T)=\left(h^{T}(k T) h^{T}(k T-d)\right)^{T} \\
& =\left(\begin{array}{ccc}
\int_{k T-d}^{k T+T-d} e^{A(k T+T-d-s)} A_{d} x(s) d s & 0 \\
0 & \int_{k T-d}^{k T+T-d} e^{A(k T+T-d-s)} A_{d} x(s-d) d s
\end{array}\right) \\
& =\int_{k T-d}^{k T+T-d} e^{A(k T+T-d-s)} \tilde{A}_{d} X(s) d s, \\
& \tilde{Z}(k T)=\left(\begin{array}{ll}
\tilde{h}^{T}(k T) & \left.\tilde{h}^{T}(k T-d)\right)^{T}
\end{array}\right. \\
& =\left(\begin{array}{ccc}
\int_{k T}^{k T+T} e^{A(k T+T-s)} G x(s) d W_{s} & 0 \\
& 0 & \int_{k T}^{k T+T} e^{A(k T+T-s)} G x(s-d) d W_{s}
\end{array}\right) \\
& =\int_{k T}^{k T+T} e^{A(k T+T-s)} \tilde{G} X(s) d W_{s} .
\end{aligned}
$$

Noting that

$$
\begin{gathered}
E\left[\|L(k T)\|^{2}\right] \leq M^{2} \beta^{2}\|B\|^{2}\|C\|^{2} E\left[\|X(k T)\|^{2}\right], \\
\|\tilde{A}\|=\|A\|, \quad\left\|\tilde{A}_{d}\right\|=\left\|A_{d}\right\|, \quad\|\tilde{G}\|=\|G\|, \quad C=\left(C_{1}, C_{2}\right), \\
\frac{E\left[\|X(t)\|^{2}\right]}{5} \leq E\left[\|X(k T)\|^{2}\right]+E\left[\left\|\int_{k T}^{t} \tilde{A} X(s) d s\right\|^{2}\right]+E\left[\|L(k T)\|^{2}\right] \\
+E\left[\left\|\int_{k T-d}^{t-d} \tilde{A}_{d} X(s) d s\right\|^{2}\right]+E\left[\left(\int_{k T}^{t} \tilde{G} x(s) d W_{s}\right)^{T}\left(\int_{k T}^{t} \tilde{G} x(s) d W_{s}\right)\right] .
\end{gathered}
$$

By Lemma 3.2, one observes that

$$
E\left[\left(\int_{k T}^{t} G x(s) d W_{s}\right)^{T}\left(\int_{k T}^{t} G x(s) d W_{s}\right)\right]=E\left[\int_{k T}^{t} x^{T}(s) G^{T} G x(s) d s\right] .
$$


Then the inequality (3.32) is reduced to

$$
\begin{aligned}
\frac{E\left[\|X(t)\|^{2}\right]}{5} \leq & K_{0} E\left[\|X(k T)\|^{2}\right]+\left\|\tilde{A}_{d}\right\|^{2} \int_{k T-d}^{t-d} E\left[\|X(s)\|^{2}\right] d s \\
& +\left(\|\tilde{A}\|^{2}+\|\tilde{G}\|^{2}\right) \int_{k T}^{t} E\left[\|X(s)\|^{2}\right] d s \\
\leq & K_{0} E\left[\|X(k T)\|^{2}\right]+\left(\|\tilde{A}\|^{2}+\|\tilde{G}\|^{2}+\left\|\tilde{A}_{d}\right\|^{2}\right) \int_{k T-d}^{t} E\left[\|X(s)\|^{2}\right] d s \\
= & K_{0} E\left[\|X(k T)\|^{2}\right]+\left(\|A\|^{2}+\|G\|^{2}+\left\|A_{d}\right\|^{2}\right) \int_{k T-d}^{t} E\left[\|X(s)\|^{2}\right] d s,
\end{aligned}
$$

where $K_{0}=1+M^{2} \beta^{2}\|B\|^{2}\|C\|^{2}$.

By the Gronwall inequality, we have

$$
\begin{aligned}
E\left[\|X(t)\|^{2}\right] & \leq 5 K_{0} E\left[\|X(k T)\|^{2}\right] e^{\left(\|A\|^{2}+\|G\|^{2}+\left\|\tilde{A}_{d}\right\|^{2}\right)(t-k T)} \\
& \leq 5 K_{0} e^{\left(\|A\|^{2}+\|G\|^{2}+\left\|A_{d}\right\|^{2}\right) T} E\left[\|X(k T)\|^{2}\right]
\end{aligned}
$$

Thus, we have

$$
\begin{aligned}
E\left[\|Z(k T)\|^{2}\right] & =E\left[\left(\int_{k T-d}^{k T+T-d} e^{A(k T+T-d-s)} \tilde{A}_{d} X(s) d s\right)^{T}\left(\int_{k T-d}^{k T+T-d} e^{A(k T+T-d-s)} \tilde{A}_{d} X(s) d s\right)\right] \\
& \leq\left\|\tilde{A}_{d}\right\|^{2} E\left[\int_{k T-d}^{k T+T-d}\left(e^{A(k T+T-d-s)}\right)^{T} e^{A(k T+T-d-s)}\|X(s)\|^{2} d s\right] \\
& \leq 5 K_{0} K_{1}\left\|A_{d}\right\|^{2} e^{\left(\|A\|^{2}+\|G\|^{2}+\left\|A_{d}\right\|^{2}\right) T} E\left[\|X(k T)\|^{2}\right] \\
& =K\left\|A_{d}\right\|^{2} e^{\left(\|G\|^{2}+\left\|A_{d}\right\|^{2}\right) T} E\left[\|X(k T)\|^{2}\right], \\
E\left[\|\tilde{Z}(k T)\|^{2}\right] & =E\left[\left(\int_{k T}^{k T+T} e^{A(k T+T-s)} \tilde{G} X(s) d W_{s}\right)^{T} \int_{k T}^{k T+T} e^{A(k T+T-s)} \tilde{G} X(s) d W_{s}\right] \\
& =E\left[\int_{k T}^{k T+T} X^{T}(s) \tilde{G}^{T}\left(e^{A(k T+T-s)}\right)^{T} e^{A(k T+T-s)} \tilde{G} X(s) d s\right] \\
& \leq\|\tilde{G}\|^{2} E\left[\int_{k T}^{k T+T}\left(e^{A(k T+T-s)}\right)^{T} e^{A(k T+T-s)}\|X(s)\|^{2} d s\right] \\
& \leq K\|G\|^{2} e^{\left(\|G\|^{2}+\left\|A_{d}\right\|^{2}\right) T} E\left[\|X(k T)\|^{2}\right],
\end{aligned}
$$


where

$$
K_{1}=\max _{s \in[0, T]}\left\|e^{A^{T} s} e^{A s}\right\|, \quad K=5 K_{0} K_{1} e^{\|A\|^{2} T} .
$$

Thus, when condition (iii) is satisfied, we have

$$
E\left[\|Z(k T)\|^{2}\right]<\mu E\left[\|X(k T)\|^{2}\right], \quad E\left[\|\tilde{Z}(k T)\|^{2}\right]<\mu E\left[\|X(k T)\|^{2}\right] .
$$

Then, we obtain

$$
\begin{aligned}
\nabla E[V(x)]= & E[V(x(k T+T))]-E[V(x(k T))] \\
& -E[V(x(k T))]+E[V(x(k T-d))] \\
\leq & -\frac{c(\mu)}{\lambda_{M}(P)} E[V(X(k T))]<0 .
\end{aligned}
$$

Therefore,

$$
E[V(X(k T+T))]<\left(1-\frac{c(\mu)}{\lambda_{M}(P)}\right) E[V(X(k T))] .
$$

Noticing that $V(X(0))=x^{T}(0) P_{1} x(0) \leq\|P\|\|x(0)\|^{2}=\|P\|\|a\|^{2}$, where $a=x(0)$ is initial state. For $t \in[k T, k T+T]$, we have

$$
\begin{aligned}
E[V(x(t))] & \leq E[V(X(k T))] \\
& \leq\left(1-\frac{c(\mu)}{\lambda_{M}(P)}\right) E[V(X(k T-T))] \\
& \leq \cdots \\
& \leq\left(1-\frac{c(\mu)}{\lambda_{M}(P)}\right)^{k} E[V(x(0))] \\
& \leq\left(1-\frac{c(\mu)}{\lambda_{M}(P)}\right)^{k}\left\|P_{1}\right\|\|x(0)\|^{2} \\
& <e^{t \ln (\Theta / \rho)}\left\|P_{1}\right\|\|x(0)\|^{2} \\
& \leq\|P\|\|a\|^{2} e^{t \ln (\Theta / \rho)}
\end{aligned}
$$

where

$$
\Theta=1-\frac{c(\mu)}{\lambda_{M}(P)}<1, \quad \rho=\min _{k}\left(T_{k}-T_{k-1}\right) .
$$


Noticing that $E[V(x(t))] \geq \lambda_{m}(P) E\|x(t)\|^{2}$, one obtains that

$$
\begin{aligned}
E\left[\|x(t)\|^{2}\right] & \leq \frac{E[V(x(t))]}{\lambda_{m}(P)} \\
& <\|a\|^{2}\left[\frac{\|P\|}{\lambda_{m}(P)}\right] e^{t \ln (\Theta / \rho)} \\
& =\psi\|a\|^{2} e^{-\beta t}
\end{aligned}
$$

where

$$
\psi=\frac{\|P\|}{\lambda_{m}(P)}>1, \quad \beta=-\ln \frac{\Theta}{\rho}>0 .
$$

Thereforce, by virtue of Definition 2.5 with $p=2$, we know that the equilibrium point of system (3.3) is uniformly exponentially stable in mean square.

Now we will proof the $p$ th moment uniformly exponentially stable in mean of system

For $p=2 q, q \geq 1$, we have

$$
\begin{aligned}
& E\left[V(X(k T+T))^{q}\right]-E\left[V(X(k T))^{q}\right] \\
& \quad=E[V(X(k T+T))-V(X(k T))]\left[V(X(k T+T))^{q-1}+\cdots+V(X(k T))^{q-1}\right] \\
& \quad \leq E\left[-\frac{c(\mu)}{\lambda_{M}(P)} V(X(k T))\right] \times\left[V(X(k T+T))^{q-1}+\cdots+V(X(k T))^{q-1}\right] \\
& \quad \leq-\frac{c(\mu)}{\lambda_{M}(P)}\left\{E\left[V(X(k T))^{q}+\cdots+V(X(k T))^{q} \times V(X(k T+T))\right]\right\} \\
& \quad \leq-\frac{c(\mu)}{\lambda_{M}(P)} E\left[V(X(k T))^{q}\right]<0 .
\end{aligned}
$$

It is obvious that

$$
E\left[V(X(k T+T))^{q}\right] \leq\left[1-\frac{c(\mu)}{\lambda_{M}(P)}\right] E\left[V(X(k T))^{q}\right] .
$$


For $t \in[k T, k T+T]$, we have

$$
\begin{aligned}
E\left[V(x(t))^{q}\right] & \leq E\left[V(X(k T))^{q}\right] \\
& \leq\left[1-\frac{c(\mu)}{\lambda_{M}(P)}\right] E\left[V(X(k T-T))^{q}\right] \\
& \leq \cdots \\
& \leq\left[1-\frac{c(\mu)}{\lambda_{M}(P)}\right]^{k} E\left[V(x(0))^{q}\right] \\
& \leq\left(1-\frac{c(\mu)}{\lambda_{M}(P)}\right)^{k}\|P\|^{q}\|x(0)\|^{2 q} \\
& <e^{t \ln (\Theta / \rho)}\|P\|^{q}\|x(0)\|^{2 q}=\|P\|^{q}\|a\|^{p} e^{t \ln (\Theta / \rho)} .
\end{aligned}
$$

Similarly, we have

$$
\begin{aligned}
E\left[\|x(t)\|^{p}\right] & \leq \frac{E\left[V(x(t))^{q}\right]}{\left[\lambda_{m}(P)\right]^{q}} \\
& <\|a\|^{p}\left[\frac{\|P\|}{\lambda_{m}(P)}\right]^{q} e^{t \ln (\Theta / \rho)} \\
& =\tilde{\psi}\|a\|^{p} e^{-\beta t},
\end{aligned}
$$

where $\widetilde{\psi}=\psi^{p / 2}>1$ is constant.

Therefore, we have shown that the trivial solution of system (3.3) is the $p$ th moment uniformly exponentially stable in mean for even integers. In the same way, the theorem is satisfied for odd integers. Hence, we conclude the proof for all $p>0$.

Remark 3.4. The upper of PWM is given by that can easily be computed and optimized. We will employ a simple procedure in a specific example in Section 4.

Remark 3.5. The $p$ th moment exponential stability considered, in this paper, the system tending to equilibrium speeds more quickly than others. The change of the status vectors as time increases will be showed in Figure 3.

Corollary 3.6. Assume that $A$ is Hurwitz stable, $C_{1} \neq 0, C_{2}=0$, namely, the output of PWM feedback system $y(t)$ linear dependences on current status vectors $x(t)$. If the parameter of PWM $M \beta$ satisfies

$$
M \beta<\frac{\lambda_{m}\left(\Gamma_{1}\right)+\sqrt{\lambda_{m}^{2}\left[\Gamma_{1}\right]+4 \lambda_{M}\left(\tilde{\Gamma}_{2}\right)}}{2 \lambda_{M}\left(\tilde{\Gamma}_{2}\right)}
$$

whenever $\|G\|$ is sufficiently small (an upper bound of $\|G\|$ has been given in the proof of Theorem 3.3), where $\Gamma_{1}=U_{1}\left(T_{k}\right) C_{1}+\left(U_{1}\left(T_{k}\right) C_{1}\right)^{T}, \widetilde{\Gamma}_{2}=C_{1}^{T}\left(G_{1}\left(\tau_{k}\right)+\widetilde{G}_{2}\left(\tau_{k}\right)+\tilde{U}_{2}^{T}\left(T_{k}\right) \tilde{U}_{2}\left(T_{k}\right)\right) C_{1}$ with 
$\tilde{G}_{2}\left(\tau_{k}\right)=W^{T}\left(\tau_{k}\right)\left(e^{-A d}\right)^{T}\left(P_{2}-I\right) e^{-A d} W\left(\tau_{k}\right), \tilde{U}_{2}\left(\tau_{k}\right)=\left(P_{2}-I\right) e^{-A d} W\left(\tau_{k}\right)$ by choosing $P$ satisfied $\left(e^{A T}\right)^{T} P e^{A T}-P=-I$.

\section{Examples}

Example 4.1. Consider the system (3.3) with one order Hurwitz stable plant described by transfer function $T(s)=1 /(s+1)$. The state space representation of this system is given by $A=-1, A_{d}=0.1, B=1, C_{1}=1, C_{2}=-1$, assuming the period $T=1$, the time delay $d=0.1$. Hence $W\left(T_{k}\right), P_{i}, G_{i}\left(T_{k}\right), U_{i}\left(T_{k}\right), i=1,2$, in Theorem 3.3 are calculated in this case as

$$
\begin{gathered}
P_{1}=\frac{1}{1-e^{-2}}, \quad P_{2}=\frac{1}{1-e^{-1.8}}, \quad W\left(T_{k}\right)=\frac{e^{2 T_{k}}-1}{T_{k}}, \\
G_{1}\left(T_{k}\right)=\left(\frac{e^{2 T_{k}}-1}{T_{k}}\right)^{2} \frac{1}{e^{2}-1}, \quad G_{2}\left(T_{k}\right)=\left(\frac{e^{2 T_{k}}-1}{T_{k}}\right)^{2} \frac{1}{e^{1.8}-1}, \\
U_{1}\left(T_{k}\right)=\frac{e^{2 T_{k}}-1}{T_{k}} \frac{1}{e^{2}-1}, \quad U_{2}\left(T_{k}\right)=\frac{e^{2 T_{k}}-1}{T_{k}} \frac{1}{e^{1.8}-1},
\end{gathered}
$$

where the pulse width $T_{k}$ satisfies $0<T_{k} \leq 0.9$, and we obtain the estimation of the upper bound of $M \beta$, that is, $M \beta \leq 0.9398$. For $M \beta \in(0,0.9398)$, we compute $\mu_{\max }=0.7$ such that for all $\mu<\mu_{\max } . c(\mu)=1-2\|\Phi\| \sqrt{\mu}-4\|P\| \mu>0$ is true. Next, we compute $\delta_{\max }=0.22$ such that $K \cdot f\left(\left\|A_{d}\right\|,\|G\|\right)<\mu_{\max }$ is true for all $\delta<\delta_{\max }$, where $K=5 K_{1}\left(1+M^{2} \beta^{2}\|B\|^{2}\|C\|^{2}\right) e^{\|A\|^{2} T}$ and $K_{1}=\max _{s \in[0, T]}\left\|e^{A^{T} s} e^{A s}\right\|$. In Figure 2, we depict the estimates of the upper bound $\delta_{\max }$ of $\|G\|$ versus $M \beta$.

We observe that $\delta_{\max }$ decreases as $M \beta$ increases. When the states are sufficiently far away from the origin so that $T_{k}=T-d$, the curvature of the curve reduces more slowly than without time-varying delays. Namely, as $M \beta$ increases, the anti-interference performance of the system (3.3) is stronger than the stochastic PWM feedback system without time-varying delays. Furthermore, as $M$ increases (for fixed $\beta$ ), the maximum $\|G\|$ allowable to ensure the $p$ th moment uniform exponential stability in mean will decrease, besides, if disturbance of the feedback system (3.3) is increased (less than $\delta_{\max }$ ), the trivial solution of system (3.3) is the $p$ th moment uniformly exponentially stable in mean by decreasing the value of $M \beta$, as shown in Figure 2.

Example 4.2. Consider PWM feedback system (3.3) with transfer function $T(s)=1 /(s+2)$ $(s+1)$.

The state space representation of this system is given by

$$
\begin{array}{cc}
A=\left(\begin{array}{cc}
-2 & 0 \\
0 & -1
\end{array}\right), \quad A_{d}=\left(\begin{array}{cc}
0.2 & 0 \\
0 & 0.1
\end{array}\right), \quad G=\left(\begin{array}{cc}
0.02 & 0 \\
0 & 0.006
\end{array}\right), \\
B=\left(\begin{array}{ll}
1 & 1
\end{array}\right)^{T}, \quad C_{1}=\left[\begin{array}{ll}
1 & 1
\end{array}\right], \quad C_{2}=\left[\begin{array}{ll}
0 & 0
\end{array}\right] .
\end{array}
$$




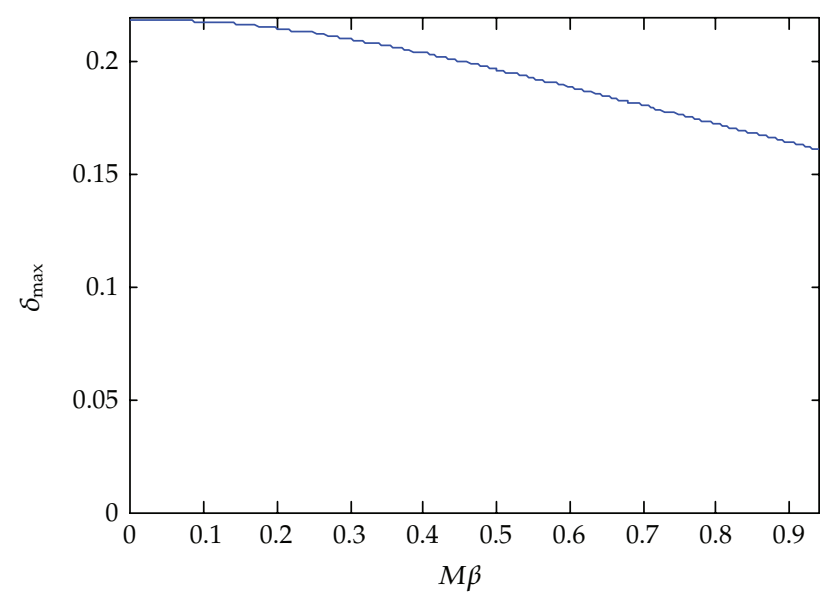

Figure 2: Upper bounds for $\|G\|$ when $M \beta \in(0,0.9398)$.

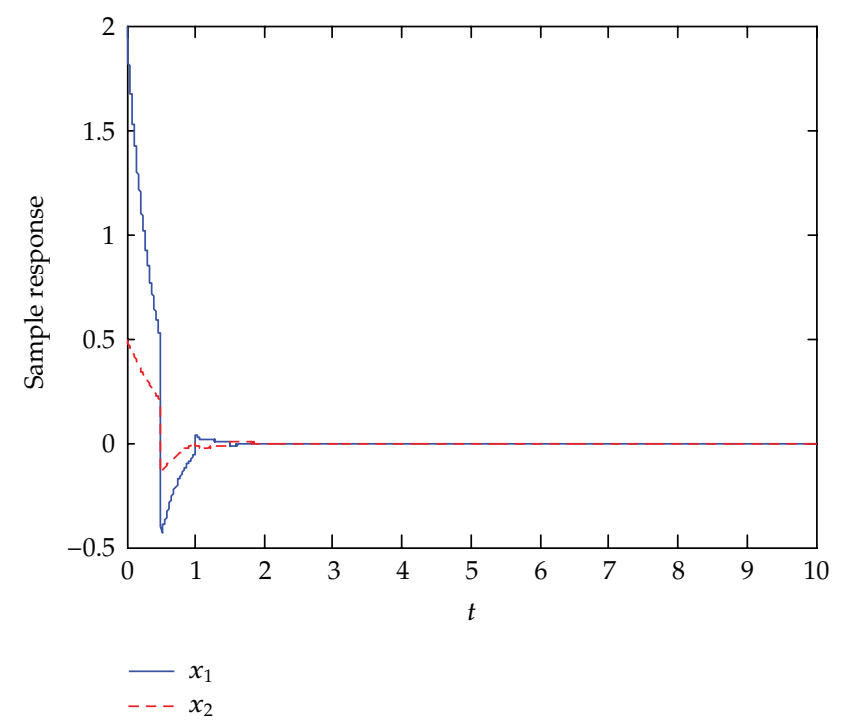

Figure 3: Sample response of PWM feedback system (3.3) in Example 4.2.

In Figure 3, we plot the sample response of $x=\left(\begin{array}{ll}x_{1} & x_{2}\end{array}\right)^{T}$ with $M=0.2, T=\beta=1, d=0.1$, and $x(0)=\left(\begin{array}{ll}2 & 0.5\end{array}\right)^{T}$. We observe the system (3.3) tending to the equilibrium point speed quickly.

\section{Conclusions}

We studied the stochastic PWM feedback systems with time-varying delays and established several Lyapunov and Lagrange criteria for the $p$ th moment exponential stability in mean, then presented an algorithm to compute the upper bound for the parameters of PWM, and finally given two numerical examples to verify the effectiveness of theoretical results. We characterized the relationship among the parameters of pulse-width modulation, time delay, 
and the coefficient of state vectors of the feedback systems and showed that when the random disturbance is sufficiently small such PWM feedback system is the $p$ th moment uniformly exponentially stable in mean provided that the upper bounds of parameters of pulse-width modulator are selected properly.

\section{Acknowledgment}

The work was supported by the Fundamental Research Funds for the Central Universities of China under Grants CDJZR10100015.

\section{References}

[1] I. Takahashi, "A new control of PWM inverter wave form for minimum loss operation of an induction motor drive," IEEE Transactions on Industry Applications, vol. 21, no. 4, pp. 580-587, 1985.

[2] S.-Y. Choe, J.-G. Lee, J.-W. Ahn, and S.-H. Baek, "Integrated modeling and control of a PEM fuel cell power system with a PWM DC/DC converter," Journal of Power Sources, vol. 164, no. 2, pp. 614-623, 2007.

[3] J.-C. Bor and C.-Y. Wu, "Realization of the CMOS pulsewidth-modulation (PWM) neural network with on-chip learning," IEEE Transactions on Circuits and Systems II, vol. 45, no. 1, pp. 96-107, 1998.

[4] Z. Hui and B. Michael, "A new partly unsymmetrical PWM technique for harmopnic compensators," in Proceedings of the 11th European Conference on Power Electronics and Applications (EPE '05), Dresdnen, Germany, September 2005.

[5] N. Bodo, M. Jones, and E. Levi, "A PWM method for seven- and nine-phase open-end winding motordrives," Mathematics and Computers in Simulation. In press.

[6] G. J. Murphy and S. H. Wu, "A stability criterion for pulse-width-modulated feedback control systems," IEEE Transactions on Automatic Control, vol. 9, pp. 434-441, 1964.

[7] A. Balestrino, A. Eisinberg, and L. Sciavicco, "A generalised approach to the stability analysis of PWM feedback control systems," Journal of the Franklin Institute, vol. 298, no. 1, pp. 45-58, 1974.

[8] R. A. Skoog, "On the stability of pulse width modulated feedback systems," IEEE Transactions on Automatic Control, vol. 13, no. 5, pp. 532-538, 1968.

[9] A. Halanay, "Positive definite kernels and stability of automatic systems," Revue Roumaine de Mathématique Pures et Appliquées, vol. 9, no. 8, pp. 751-765, 1964 (Russian).

[10] V. M. Popov, "On absolute stability of nonlinear systems of automatic regulation," Avtomatika $i$ Telemekhanika, vol. 22, no. 8, pp. 961-979, 1961.

[11] S. C. Gupta and E. I. Jury, "Statistical study of pulse-width modulated control systems," Journal of the Franklin Institute, vol. 273, no. 4, pp. 292-321, 1962.

[12] L. Hou and A. N. Michel, "Moment stability of pulse-width-modulated feedback systems subjected to random disturbances," in Proceedings of the 39th IEEE Confernce on Decision and Control, pp. 2343-2348, Sydney, Australia, December 2000.

[13] L. Hou and A. N. Michel, "Moment stability of discontinuous stochastic dynamical systems," in Proceedings of the Americal Control Conference, vol. 6, pp. 3807-3811, Chicago, Ill, USA, June 2000.

[14] S. Boyd, L. EI Ghaoui, E. Feron, and V. Balakrishnan, Linear Matrix Inequalities in System and Control Theory, SIAM Studies in Applied Mathematics, SIAM, Philadelphia, Pa, USA, 1994.

[15] P. Cheng, F. Deng, and Y. Peng, "Robust exponential stability and delayed-state-feedback stabilization of uncertain impulsive stochastic systems with time-varying delay," Communications in Nonlinear Science and Numerical Simulation, vol. 17, no. 12, pp. 4740-4752, 2012.

[16] W. Su and Y. Chen, "Global robust exponential stability analysis for stochastic interval neural networks with time-varying delays," Communications in Nonlinear Science and Numerical Simulation, vol. 14, no. 5, pp. 2293-2300, 2009.

[17] Y. Sun and J. Cao, " $p$ th moment exponential stability of stochastic recurrent neural networks with time-varying delays," Nonlinear Analysis: Real World Applications, vol. 8, no. 4, pp. 1171-1185, 2007.

[18] C. Li, T. Huang, G. Feng, and G. Chen, "Exponential stability of time-controlled switching systems with time delay," Journal of the Franklin Institute, vol. 349, no. 1, pp. 216-233, 2012.

[19] H. Huang and G. Feng, "Delay-dependent stability for uncertain stochastic neural networks with time-varying delay," Physica A, vol. 381, no. 1-2, pp. 93-103, 2007. 
[20] C. Li, C. Li, X. Liao, and T. Huang, "Impulsive effects on stability of high-order BAM neural networks with time delays," Neurocomputing, vol. 74, no. 10, pp. 1541-1550, 2011.

[21] J. Tian, S. Zhong, and Y. Wang, "Improved exponential stability criteria for neural networks with time-varying delays," Neurocomputing, vol. 97, no. 15, pp. 164-173, 2012.

[22] Z. Wu, H. Su, J. Chu, and W. Zhou, "New results on robust exponential stability for discrete recurrent neural networks with time-varying delays," Neurocomputing, vol. 72, no. 13-15, pp. 3337-3342, 2009. 


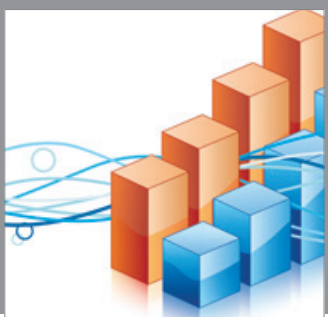

Advances in

Operations Research

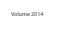

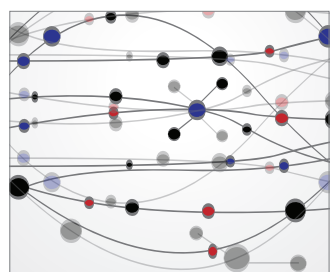

\section{The Scientific} World Journal
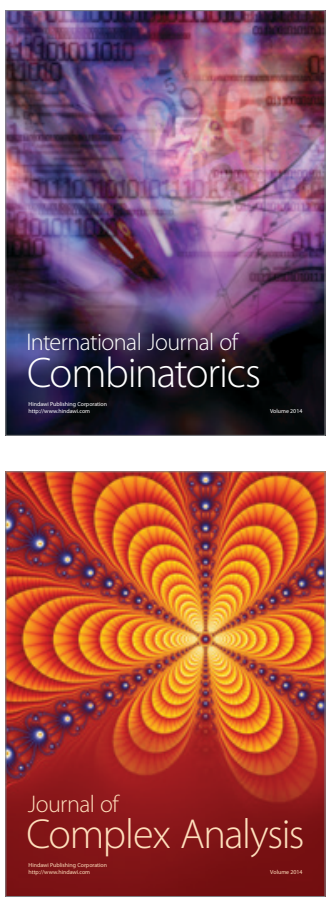

International Journal of

Mathematics and

Mathematical

Sciences
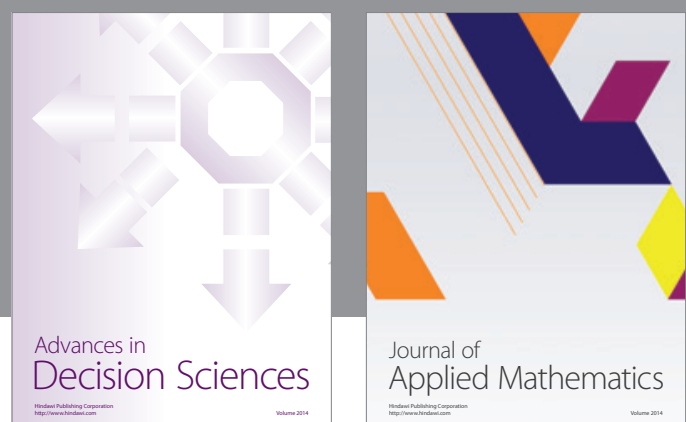

Journal of

Applied Mathematics
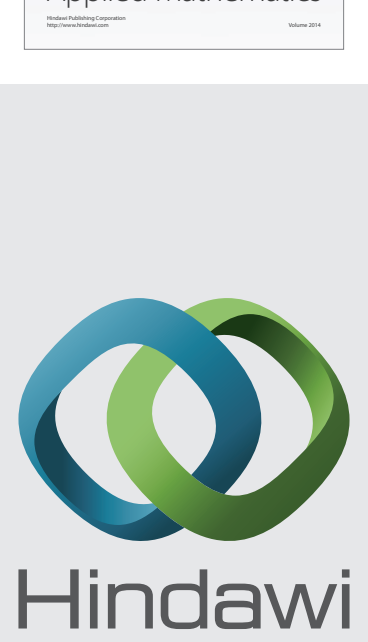

Submit your manuscripts at http://www.hindawi.com
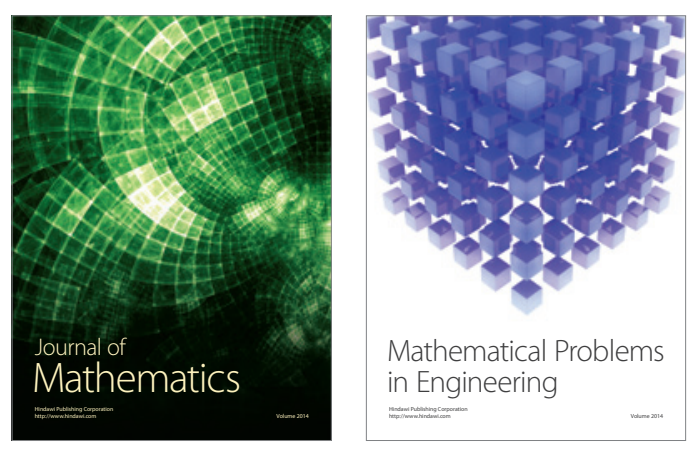

Mathematical Problems in Engineering
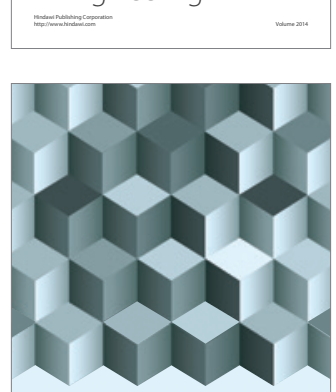

Journal of

Function Spaces
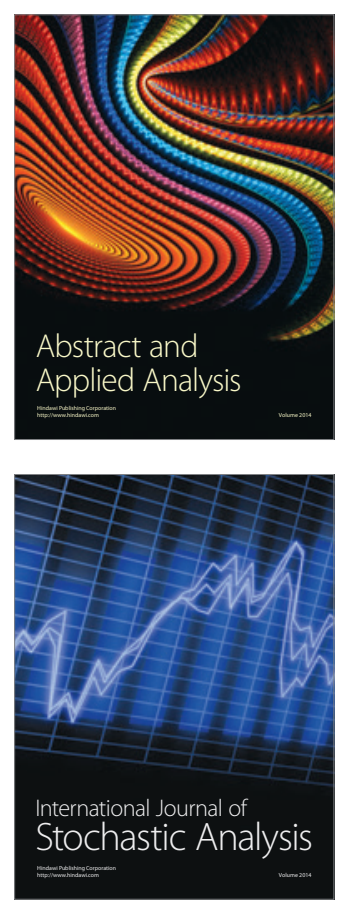

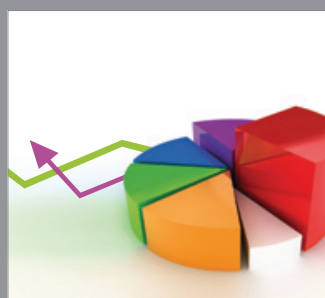

ournal of

Probability and Statistics

Promensencen
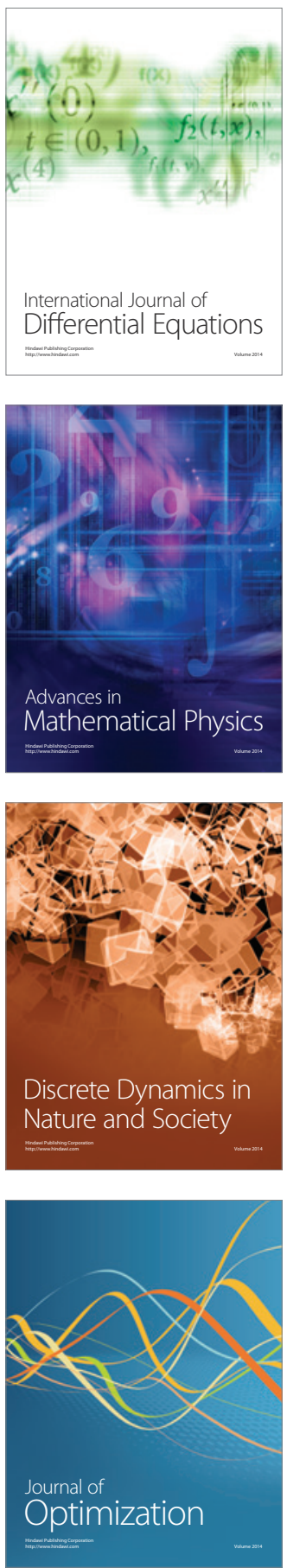